\title{
Optical-resolution photoacoustic microscopy of angiogenesis in a transgenic mouse model
}

Song $\mathrm{Hu}$, Sunday Oladipupo, Junjie Yao, Andrea C. Santeford, Konstantin Maslov, et al.

Song Hu, Sunday Oladipupo, Junjie Yao, Andrea C. Santeford, Konstantin Maslov, Joanna Kovalski, Jeffrey M. Arbeit, Lihong V. Wang, "Opticalresolution photoacoustic microscopy of angiogenesis in a transgenic mouse model," Proc. SPIE 7564, Photons Plus Ultrasound: Imaging and Sensing 2010, 756406 (23 February 2010); doi: 10.1117/12.841177

SPIE. Event: SPIE BiOS, 2010, San Francisco, California, United States 


\title{
Optical-resolution photoacoustic microscopy of angiogenesis in a transgenic mouse model
}

\author{
Song $\mathrm{Hu}^{1}$, Sunday Oladipupo ${ }^{2}$, Junjie Yao ${ }^{1}$, Andrea C. Santeford ${ }^{2}$, Konstantin Maslov ${ }^{1}$, Joanna \\ Kovalski ${ }^{2}$, Jeffrey M. Arbeit ${ }^{2,3, *}$ and Lihong V. Wang ${ }^{1, *}$ \\ ${ }^{1}$ Optical Imaging Laboratory, Department of Biomedical Engineering, Washington University in St. \\ Louis, St. Louis, Missouri, USA 63130 \\ ${ }^{2}$ Urology Division, Department of Surgery, Washington University School of Medicine, St. Louis, \\ Missouri, USA 63110 \\ ${ }^{3}$ Siteman Cancer Center, Washington University School of Medicine, St. Louis, Missouri, USA \\ 63110 \\ *Correspondence should be addressed to arbeitj@wustl.edu (animal model) and \\ lhwang@biomed.wustl.edu (photoacoustics).
}

\begin{abstract}
A major obstacle in studying angiogenesis is the inability to noninvasively image neovascular development in an individual animal. We applied optical-resolution photoacoustic microscopy (OR-PAM) to determine the kinetics of hypoxia-inducible factor-1 (HIF-1)-mediated angiogenesis in a transgenic mouse model. During continuous 30-day activation of HIF-1 $\alpha$, we used OR-PAM to monitor alterations in microvasculature in transgenic mice compared to nontransgenic mice. OR-PAM has demonstrated the potential to precisely monitor antiangiogenic therapy of human cancers, allowing for rapid determinations of therapeutic efficacy or resistance.
\end{abstract}

Keywords: Optical-resolution photoacoustic microscopy, chronic imaging, angiogenesis, HIF-1 $\alpha$.

\section{INTRODUCTION}

A major challenge in angiogenesis research is how to monitor the temporal aspects of the angiogenic process in intact animals. Conventional ex vivo histology analysis requires animal termination; therefore, only a single time point can be evaluated per animal. In vivo optical microscopy, such as intravital microscopy, confocal microscopy, or multiphoton microscopy, allows repetitive imaging; however, the required angiographic contrast agents may alter the behavior of the underlying microcirculation, and the window preparation has a limited life time. Here, we applied optical-resolution photoacoustic microscopy (OR-PAM), a newly developed optical-ultrasonic hybrid technique capable of imaging the intact microvessels down to single capillaries in vivo ${ }^{1-3}$, to monitor the temporal aspects of angiogenesis in a transgenic mouse model.

\section{METHODS AND MATERIALS}

\subsection{System description}

In our OR-PAM system (Fig. 1), a wavelength-tunable laser system, consisting of a dye laser an Nd:YLF pump laser, is used as the excitation source. The laser beam coming out of the dye laser is attenuated and spatially filtered by a pinhole. The reshaped laser beam is then focused to a diffraction-limited optical focal spot by a microscope objective. The optical illumination and the ultrasonic detection are configured coaxially and confocally using a home-made acoustic-optical beam splitter ${ }^{4}$. In this acoustic-optical confocal configuration, the lateral resolution is determined by the diffractionlimited optical focusing to achieve $5-\mu \mathrm{m}$ lateral resolution.

Photons Plus Ultrasound: Imaging and Sensing 2010, edited by Alexander A. Oraevsky, Lihong V. Wang, Proc. of SPIE Vol. 7564,756406 - (C) 2010 SPIE · CCC code: 1605-7422/10/\$18 - doi: 10.1117/12.841177 


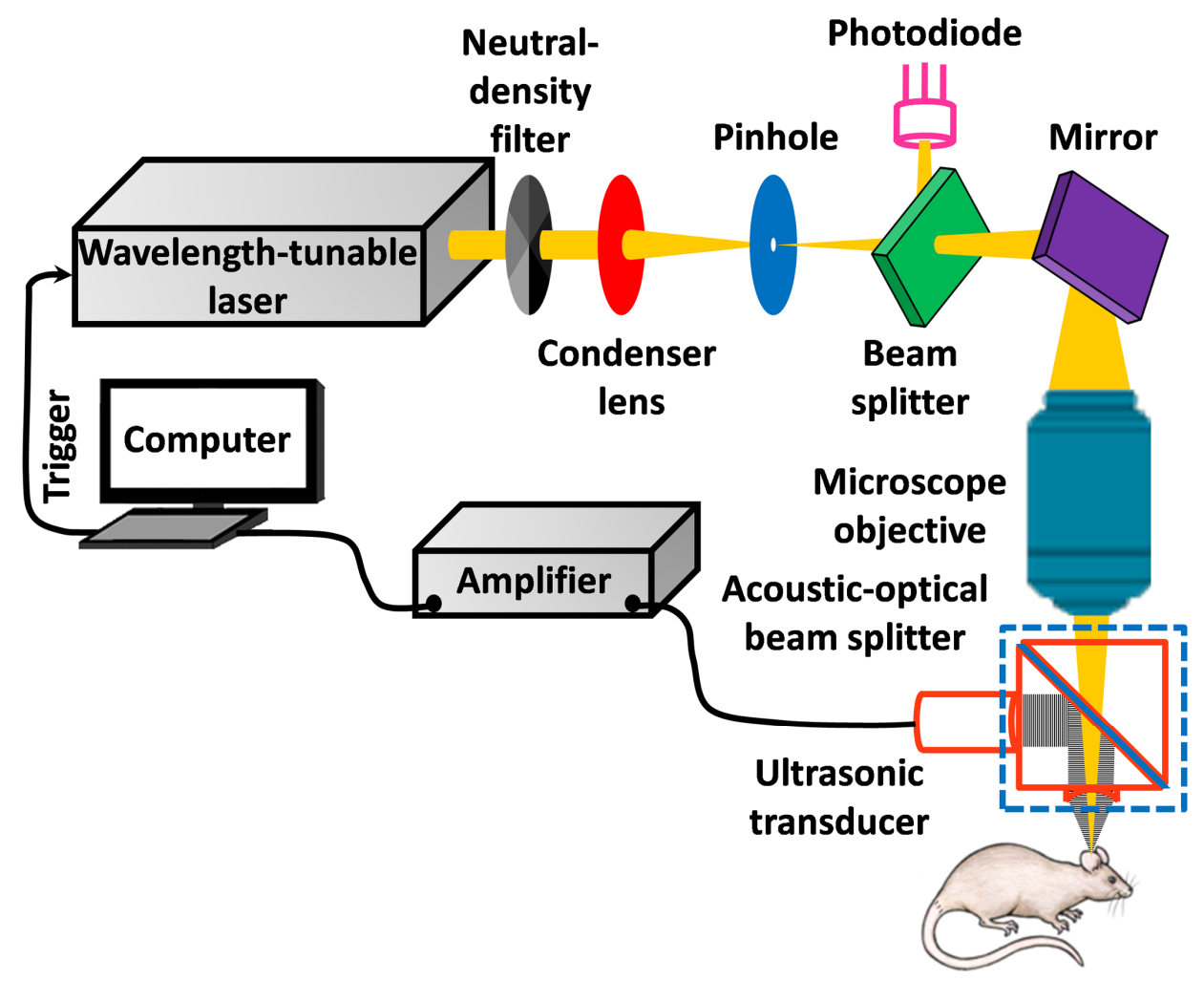

Figure 1. Schematic of the OR-PAM system ${ }^{3,5}$.

\subsection{Animal preparation}

All experimental animal procedures were carried out in conformance with the laboratory animal protocol approved by the School of Medicine Animal Studies Committee of Washington University in St. Louis.

One day before each chronic monitoring experiment on day 0 , the hair of the experimental mouse ear was gently removed with hair-removing lotion. At each time point (day 0, 1, 3, 14, and 30), a dose of $87 \mathrm{mg} / \mathrm{kg}$ ketamine and 13 $\mathrm{mg} / \mathrm{kg}$ xylazine was administered intraperitoneally to anesthetize the animal right before transferring it to a stereotaxic imaging stage. Throughout the experiment, anesthesia was maintained using vaporized isoflurane $(1.0-1.5 \%$ isoflurane with an airflow rate of $1 \mathrm{~L} / \mathrm{min}$ ), and the body temperature of the animal was maintained at $37^{\circ} \mathrm{C}$ with a temperature controlled heating pad. After the experiment, the imaged ear was cleaned gently with ultrapure water. At the end of the chronic study on each individual animal, it was euthanized by an intraperitoneal administration of pentobarbital at a dosage of $100 \mathrm{mg} / \mathrm{kg}$.

\section{RESULTS AND DISCUSSION}

Chronic OR-PAM monitoring of individual transgenic mouse (Fig. 2) demonstrated progressive and ultimately a marked increase in microvessel density during continual epithelial HIF-1 $\alpha$ induction. In contrast to the changes detected in the transgenic mouse, a control experiment on a nontransgenic mouse evidenced no noticeable change in blood vessel density (Fig. 3). 


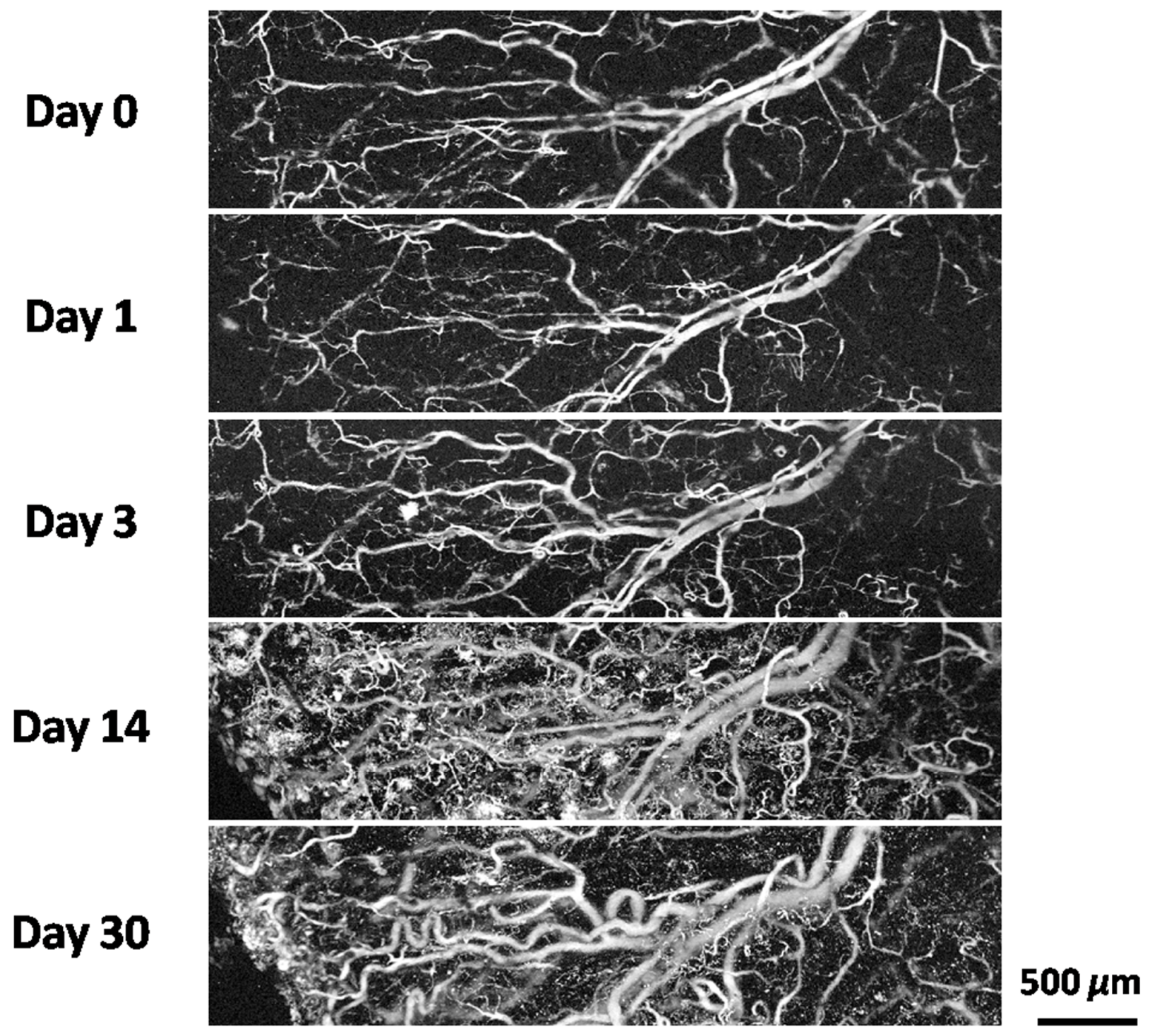

Figure 2. Chronic monitoring of HIF-mediated angiogenesis in a transgenic mouse using OR-PAM. 


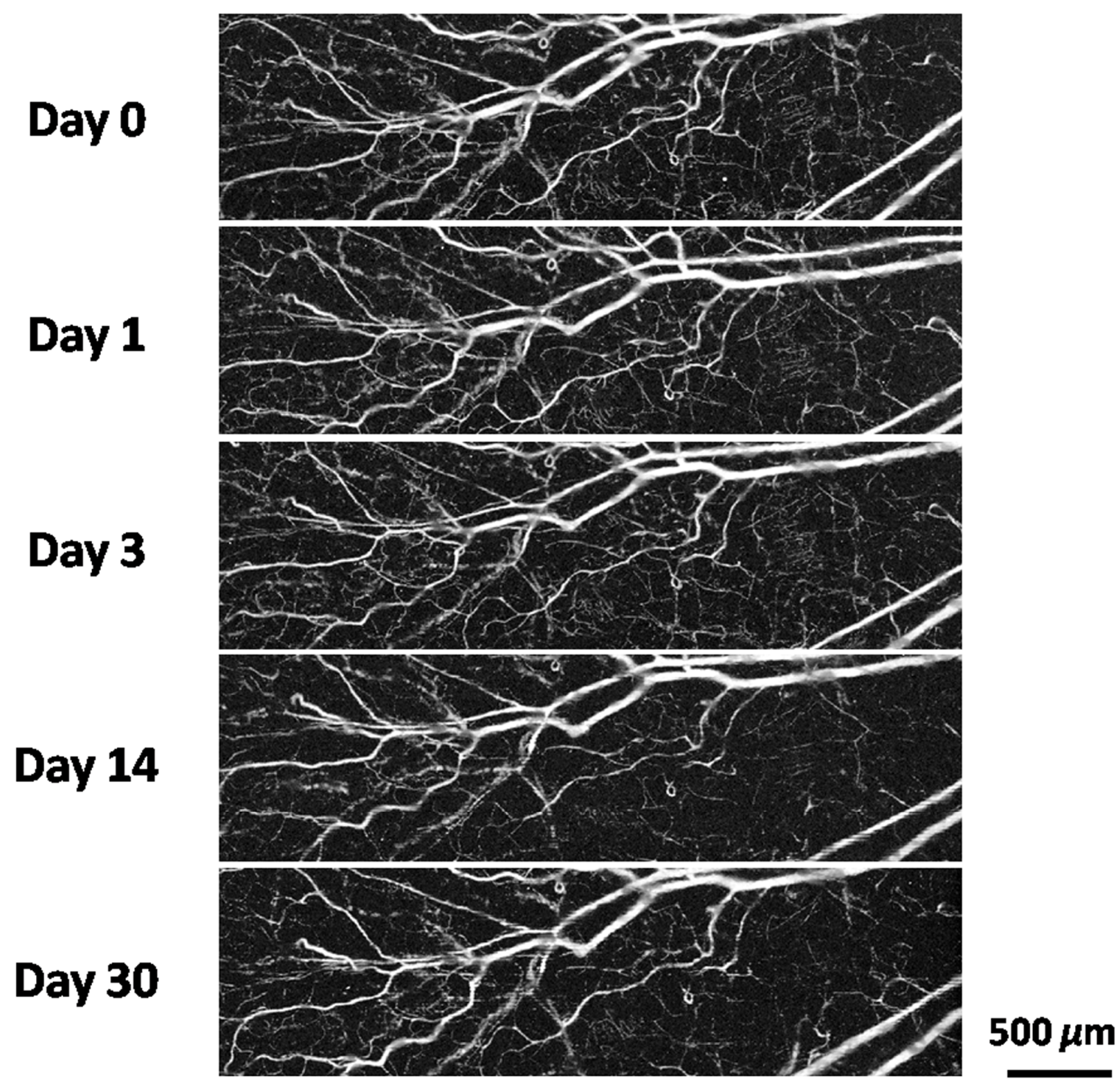

Figure 3. Chronic monitoring of a non-transgenic mouse using OR-PAM.

\section{CONCLUSIONS}

OR-PAM, capable of imaging microvasculature noninvasively and longitudinally, is a valuable tool for chronic study of microhemodynamics. In this work, we successfully demonstrated OR-PAM as a powerful technique for high-resolution functional imaging and chronic monitoring of angiogenesis in a transgenic mouse model. 


\section{ACKNOWLEDGEMENTS}

The authors thank Rebecca Sohn for mouse husbandry, Rick Bruick for the gift of the HIF-1 $\alpha^{\text {PPN }}$ plasmid for construction of the TRE-HIF- $1 \alpha^{\text {PPN }}$ transgenic mice, Adam Glick for the gift of the K5-rtTa transgenic mice, and ImClone for the generous gift of the DC101, and MF1 antibodies, Fulu Liu, Alyssa Gregory, Kyle Eash and Dan Link for help with bone marrow transplantation and advice on AMD3100 experiments. This work was supported by NCI R01CA90722, National Institutes of Health Grants R01 EB000712, R01 NS46214, R01 EB008085, and U54 CA136398. L.V.W. has financial interest in Microphotoacoustics, Inc. and Endra, Inc., which, however, did not support this work.

\section{REFERENCES}

1. S. Hu, K. Maslov, V. Tsytsarev and L. V. Wang, "Functional transcranial brain imaging by optical-resolution photoacoustic microscopy," J. Biomed. Opt. 14(4), 040503 (2009)

2. S. Hu, K. Maslov and L. V. Wang, "Noninvasive label-free imaging of microhemodynamics by opticalresolution photoacoustic microscopy," Opt. Express 17(9), 7688-7693 (2009)

3. $\quad$ S. Hu, B. Rao, K. Maslov and L. V. Wang, "Label-free Photoacoustic Ophthalmic Angiography," Opt. Lett. 35(1), 1-3 (2010)

4. S. Hu, K. Maslov and L. V. Wang, "In vivo functional chronic imaging of a small animal model using opticalresolution photoacoustic microscopy," Med. Phys. 36(6), 2320-2323 (2009)

5. S. Hu and L. V. Wang, "Photoacoustic imaging and characterization of the microvasculature," J. Biomed. Opt. 15(1), $011101(2010)$ 